\title{
A ANÁLISE DE ESPÉCIES DE AVES COMO INDICADORES AMBIENTAIS NO AMBIENTE URBANO DO MUNICÍPIO DE REGENTE FEIJO-SP.
}

\author{
Luiz Waldemar de Oliveira
}

Universidade do Oeste Paulista - UNOESTE, Ciências Biológicas, Presidente Prudente - SP. E-mail: oliveira@unoeste.br

\section{RESUMO}

Espécies de aves como Patagioenas picazuro, Zenaida auriculata, Pitangus sulphuratus, Megarynchus pitangua, Molothrus bonariensis, Psittacara leucophtalmus, Columbina talpacoti, Tyrannus malancholicus, Vanelus chilensis habitam áreas urbanas, convivendo com espécies exóticas como Passer domesticus e Columba livia. A pesquisa foi desenvolvida em praças da cidade de Regente Feijó. As observações foram feitas das 7:00 às 11:00 nos meses de janeiro a abril de 2014. Foram selecionadas 11 espécies de aves, estas relatadas por vários autores como as mais comuns em ambientes urbanizados. As espécie de aves com maior número de frequência de visualizações em Regente Feijó foi Passer domesticus (206 indivíduos; média=12.87/dia analisado) Psittacara leucophtalmus (184 indivíduos; média= 11.5/dia analisado). Estas são as espécies melhores apontadas como bioindicadores de qualidade ambiental.

Palavras-chave: área urbana, bioindicadores, qualidade, ambiental.

\section{ANALYSIS OF BIRDS SPECIES AS ENVIRONMENTAL INDICATORS IN THE URBAN ENVIRONMENT OF REGENTE FEIJÓ-SP}

\begin{abstract}
Bird species such as Patagioenas picazuro, Zenaida auriculata, Pitangus sulphuratus, Megarynchus pitangua, Molothrus bonariensis, Psittacara leucophtalmus, Columbina talpacoti, Tyrannus malancholicus, Vanelus chilensis inhabit urban areas, living with exotic species such as Passer domesticus and Columba livia. The research was conducted in the city of squares of Regente Feijó. The observations were made from 7:00 to $11: 00$ in the months from January to April 2014. 11 species of birds, those reported by several authors as the most common in urbanized environments were selected. The species of birds with more frequency previews Regente Feijó was Passer domesticus (206 individuals, mean $=12.87$ / day analyzed) Psittacara leucophtalmus (184 individuals; mean $=11.5 /$ day analysis). These are the best species identified as indicators of environmental quality.
\end{abstract}

Keywords: urban area, biomarkers, quality, environmental.

\section{INTRODUÇÃO}

A classe Aves (Chordata:Vertebrata) inclui mais de 9.000 espécies distribuídas em todo o mundo e se constitui no grupo mais homogêneo de vertebrados. As aves podem ser encontradas nos ambientes mais variados, inclusive nas proximidades de residências e centros urbanos. Podem ser descritas três categorias de aves encontradas em ambientes urbanos: aves que já se 
encontravam no ambiente antes de ser modificado; aves invasoras e as introduzidas ou exóticas (SICK, 2001).

De acordo com a CBRO (2014) existem atualmente no Brasil 1.901 espécies de aves, entre residentes e visitantes. Isso corresponde a mais da metade das espécies de aves registradas para a América do Sul (http://www.cbro.org.br/CBRO/num.htm), em torno de $20 \%$ do planeta.

A presença das aves no ambiente sugere que o mesmo pode ser saudável e funcional (DONNELY; MARZLUFF, 2006; BRUN; LINK; BRUN, 2007; PAETZOLD; QUEROL, 2008). As aves constituem um dos grupos zoológicos mais distintos e bem estudados em qualquer ambiente, podendo ser facilmente apontadas como bioindicadores de alterações ambientais (EMLEN, 1974; VERNER, 1981; AMÂNCIO; SOUZA; MELO 2008). Além disso, têm representantes em quase todos os níveis tróficos e utilizam uma grande variedade de habitat, sobretudo os terrestres. Em termos funcionais, as aves podem contribuir em diversos aspectos, desde o controle biológico de pragas (formigas, carrapatos, ratos, cobras) à serviços ecológicos como a polinização de flores e dispersão de sementes.

Segundo Sick (2001) a grande interferência humana nos ambientes naturais, para a realização agropecuária, condomínios e/ou distritos industriais, reduz o habitat, tornando-os pequenos demais para abrigarem grande diversidade de espécies animais que necessitam de um local amplo, sendo impossível preservar uma rica avifauna.

Estudos recentes objetivaram verificar a permanência das espécies em ambientes modificados pelo homem, tais como cidades (SCHERER; SCHERER; PETRY; TEIXEIRA 2006; BRUN; LINK; BRUN, 2007; TORGA; FRANCHIN; MARÇAL JÚNIOR, 2007; JEBAI et al, 2009) e Campus universitários (campi) (MONTEIRO; BRANDÃO, 1995; PHILIPPSEN; BENEDITO; ZAWADZKI, 2010) que são áreas geralmente urbanizadas ou representam zonas suburbanas, compreendendo ou não fragmentos de vegetação natural e/ou exótica (VOTTO; GOMES-JUNIOR; BUGONI; PEREIRAJUNIOR, 2006).

Este trabalho teve como objetivo identificar as espécies de aves mais ajustadas ao ambiente urbano da cidade de Regente Feijó. Ainda não há levantamentos da avifauna nessa localidade. As informações aqui relatadas poderão contribuir para uma melhor caracterização da avifauna local, e um planejamento urbano efetivo, bem como para o entendimento de relações entre o homem e a natureza, tanto do ponto de vista estético, cultural como recreativo. 
MATERIAIS E MÉTODOS.

O Município de Regente Feijó possui uma área de 265,087 km². Está localizado no oeste paulista (2213'17" , 51ำ'10"; ;altitude de 504 metros). Sua população é atualmente estimada em 18.042 habitantes.

Quatro praças foram selecionadas para a realização deste estudo: 1) praça do bairro São Sebastião; 2) praça do êxito; 3) praça de skate; 4) praça Dr. Thomé da Rocha de Oliveira. Estas distam no mínimo $1 \mathrm{~km}$ umas das outras. Devido a isso, os problemas de sobreposições na coletas dos dados foram minimizados.

Foram realizados 16 dias de observações, sempre com início às 07:00h e com término às 11:00hs, com sessões de aproximadamente uma hora em cada ponto de observação, alternandose a sequência de início de observação em cada um dos dias trabalhados. Foram realizadas quatro repetições para cada sequência, totalizando um esforço amostral de 64 horas entre meses de janeiro, e maio de 2014.

Para identificação das aves foi utilizado binóculos Power Pack Bem-1050 (10 x 50), quando necessário, as imagens foram registradas através de câmera semi-profissional (SONY, modelo CYBERSHOT) e posteriormente identificadas através de guias (SICK, 2001; ENDRIGO, 2010; DEVELEY E ENDRIGO, 2011). A ordem taxonômica segue a proposta pelo CBRO (2014) Foram contados todos os indivíduos de cada espécie observados em cada ponto nos horários estabelecidos, sendo computados os indivíduos que pousavam nas dependências das praças para forragear, descansar ou cortejar. Indivíduos em vôo, na categoria percurso (indivíduos que apenas "passavam" pelos pontos), não foram considerados, este procedimento foi adotado por Torga; Franchin e Marçal Júnior (2007).

\section{RESULTADOS}

Após um levantamento preliminar, determinou-se que durante o período de estudo, 11 espécies diferentes de aves (tabela 1), consideradas residentes e de alta incidência e frequência em ambientes urbanos e alterados tiveram as suas frequências analisadas. 
Tabela 1. Espécies de aves que tiveram suas frequências observadas.

\begin{tabular}{ll}
\hline Espécies & NOME VULGAR \\
\hline Columba livia & Pombo-doméstico \\
\hline Columbina talpacoti & Rolinha-roxa \\
\hline Patagioenas picazuro & Pomba-asa-Branca (pombão) \\
\hline Zenaida auriculata & Avoante (pomba-de-bando) \\
\hline Pitangus sulphuratus & Bem-te-ví \\
\hline Megarynchus pitanga & Bem-te-ví-do-bico-chato \\
\hline Tyrannus melancholicus & Suiriri \\
\hline Passer domesticus & Pardal \\
\hline Molothrus bonariensis & Chopim \\
\hline Psittacara leucophtalmus & Periquitão-maracanã \\
\hline Venelus chilensis & Quero-quero \\
\hline
\end{tabular}

Os resultados obtidos encontram-se dispostos nas figuras 1, 2, 3, 4, 5 e 6, onde são apresentadas a quantidade de aves observadas por espécie nos respectivos horários de observação de acordo com o ponto estabelecido.

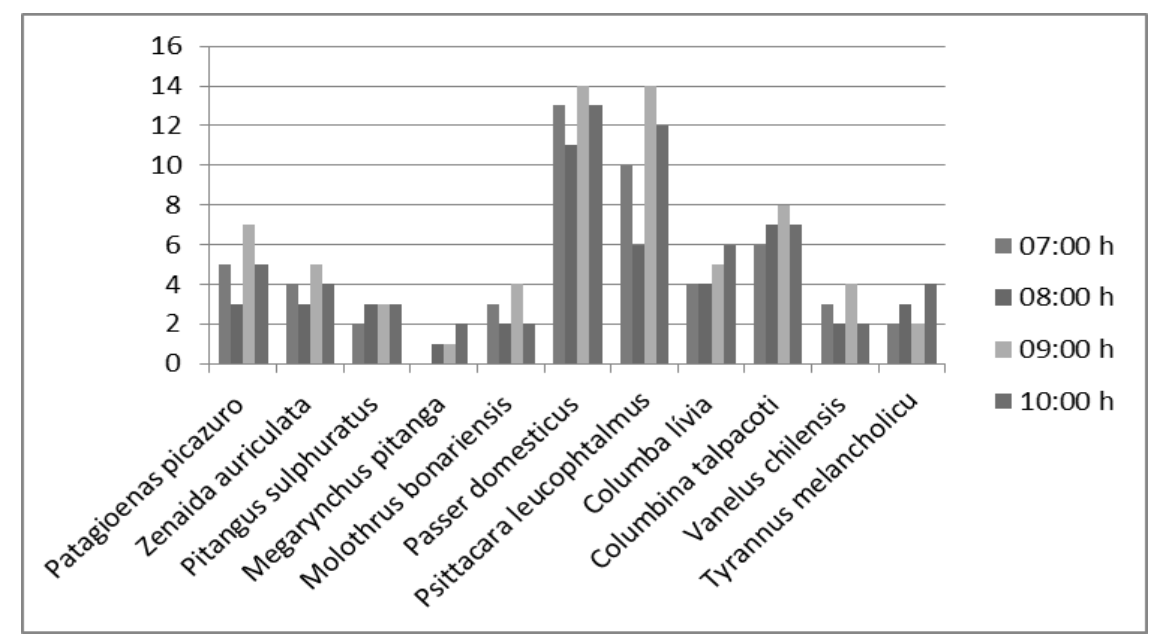

Figura 1. Quantidade total de avistamentos de cada espécie durante o período de estudos na praça do bairro São Sebastião. 


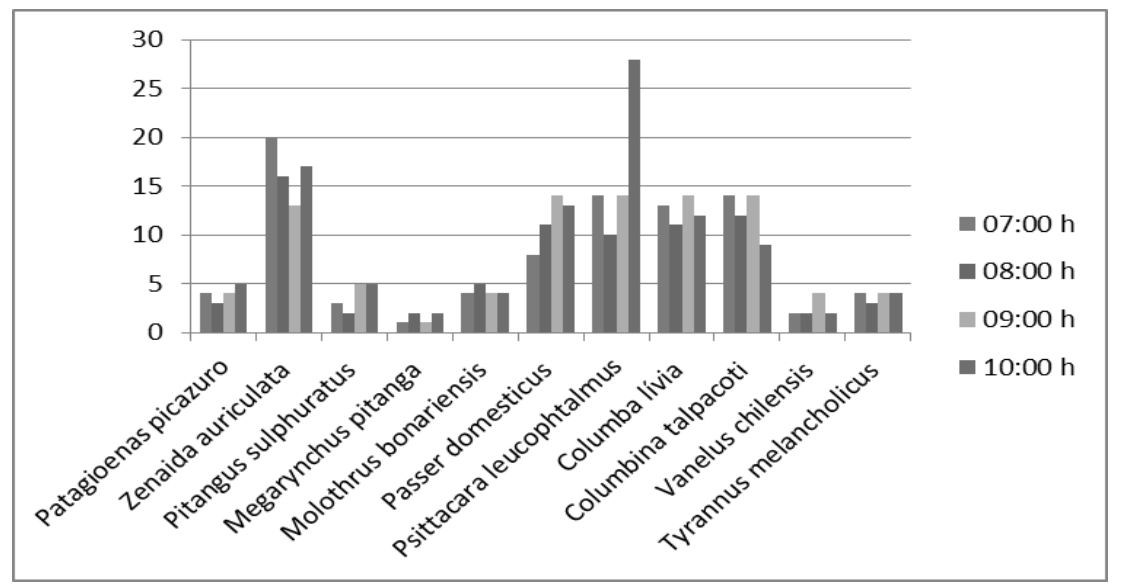

Figura 2. Quantidade total de avistamentos de cada espécie durante o período de estudos na praça do êxito.

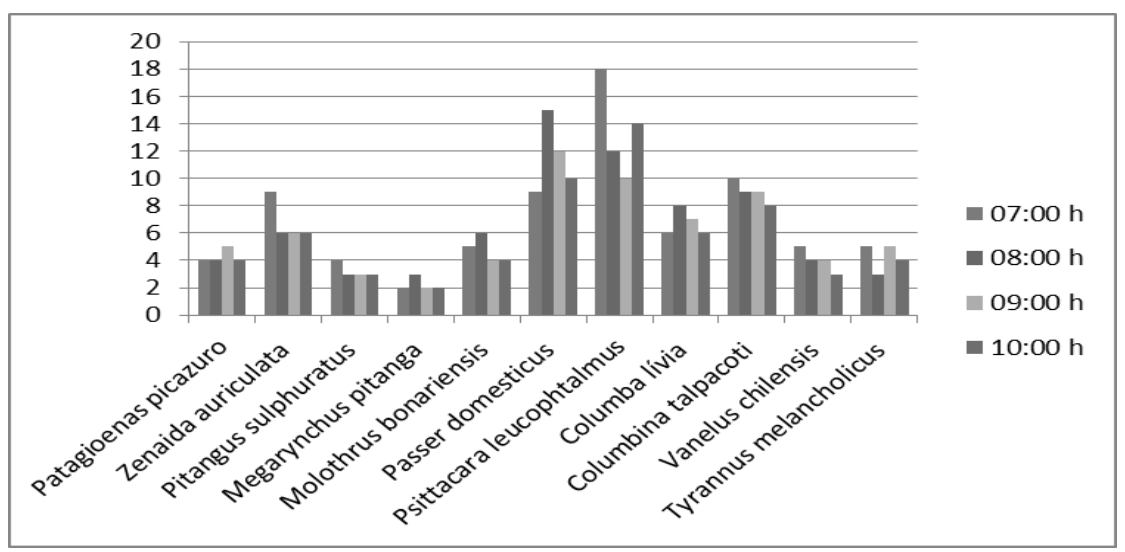

Figura 3. Quantidade total de avistamentos de cada espécie durante o período de estudos na praça de skate.

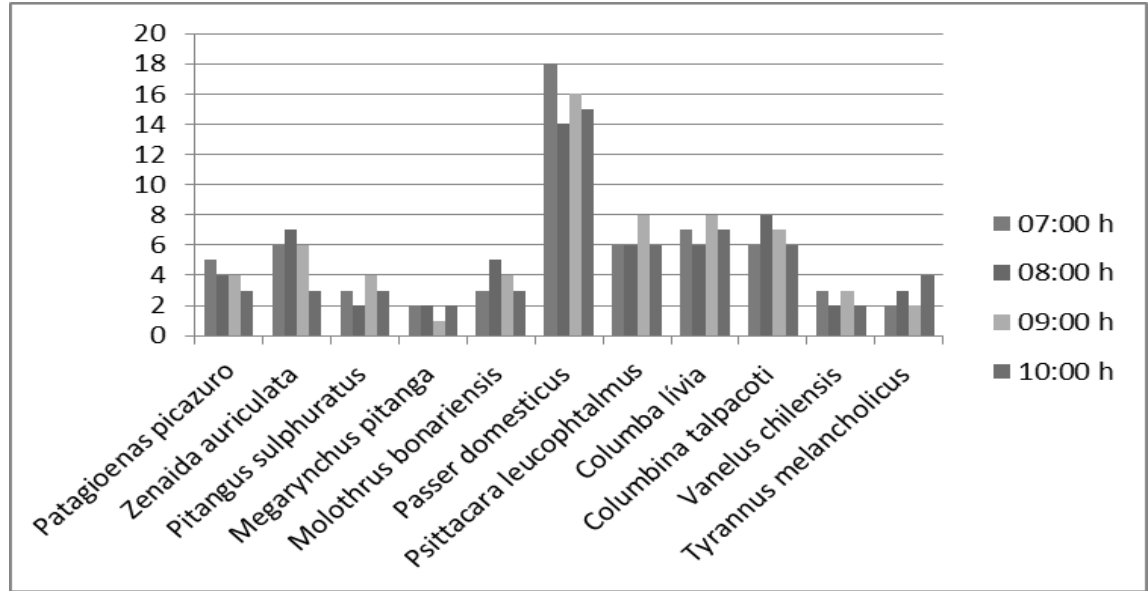

Figura 4. Quantidade total de avistamentos de cada espécie durante o período de estudos na praça Dr. Thomé da Rocha de Oliveira. 


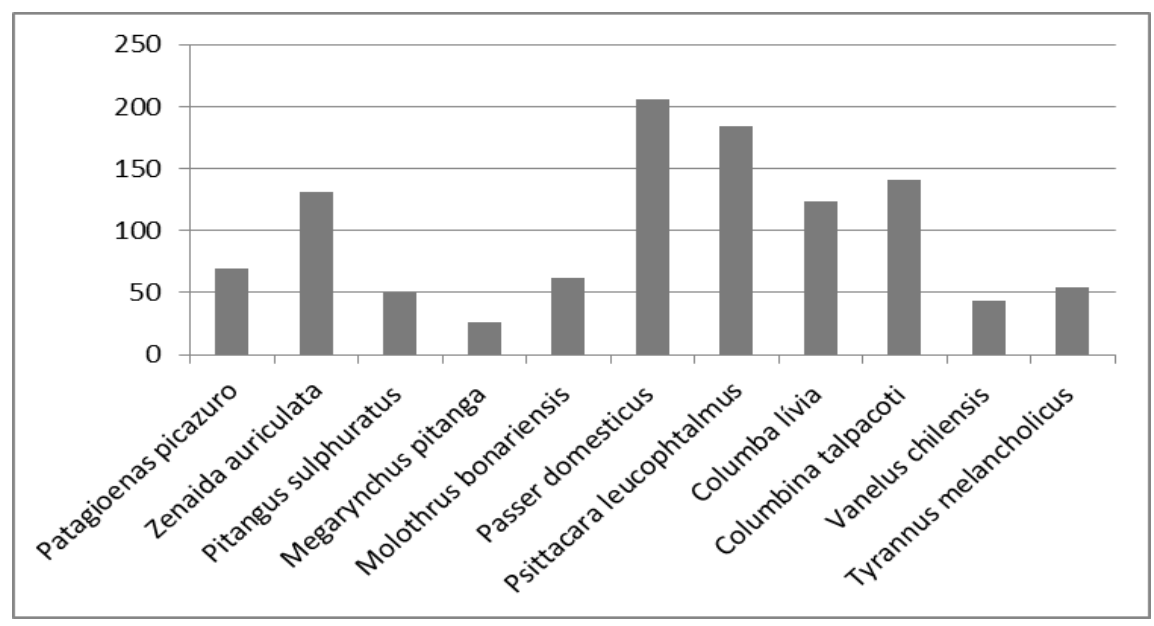

Figura 5. Total de indivíduos avistados de cada espécie, levando-se em consideração as quatro praças analisadas.

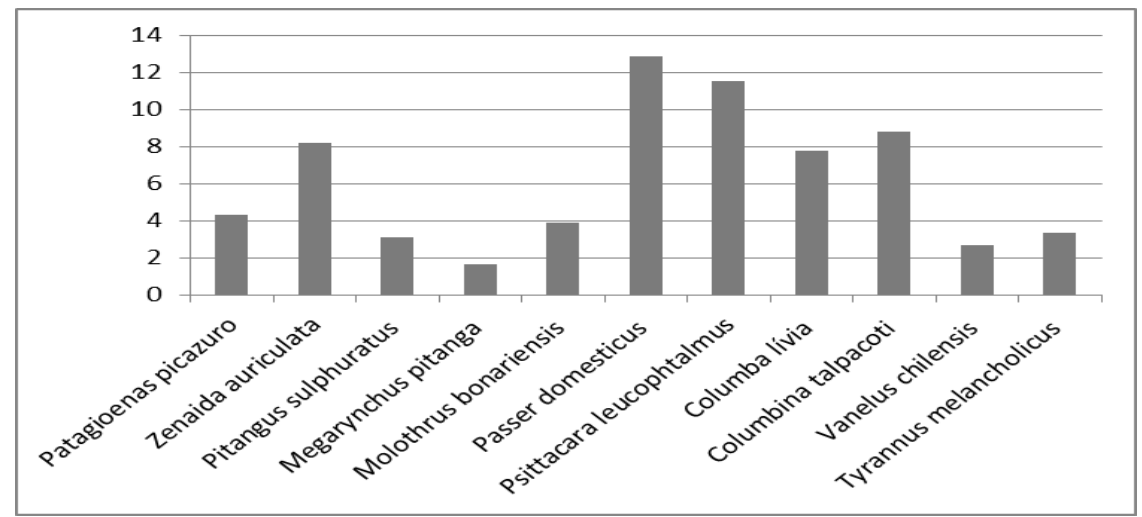

Figura 6. Média de visualizações de cada espécie/dia, levando-se em consideração as quatro praças analisadas.

As espécies de aves com maior número de frequência de visualizações em Regente Feijó foi Passer domesticus (206 indivíduos; média=12.87/dia analisado) Psittacara leucophtalmus (184 indivíduos; média= 11.5/dia analisado). Estas são as espécies melhores apontadas como bioindicadores de qualidade ambiental.

\section{DISCUSSÃO}

As espécies mais abundantes dentre as analisadas, Passer domesticus (206 indivíduos; média=12.87/dia analisado), Psittacara leucophtalmus (184 indivíduos; média= 11.5/dia analisado), Columbina talpacoti (141 indivíduos; média=8.81/dia analisado), Zenaida auriculata (131 indivíduos; média=8.18/dia analisado) e Columba lívia (124 indivíduos; média=7,75/dia analisado) podem ser consideradas as mais adaptadas ao ambiente urbano estudado. As praças utilizadas para o presente estudo dispõem de uma razoável arborização, contendo espécies 
arbóreas exóticas e nativas, este fato contribui para atrair a avifauna, aparentemente favorecendo espécies menos exigentes quanto à qualidade ambiental e à oferta de recursos (TORGA; FRANCHIN; MARÇAL JÚNIOR, 2007).

As aves são consideradas por alguns autores como bioindicadoras de qualidade ambiental urbana (DONNELY; MARZLUFF, 2006), podendo ser consideradas um bioindicador negativo quando a espécie observada possui uma população muito grande em um ambiente pouco favorável ao estabelecimento da maior parte das espécies (EMLEN, 1974; AMÂNCIO; SOUZA E MELO, 2008; PAETZOLD; QUEROL, 2008), ou como bioindicador positivo quando indivíduos de espécies mais comuns aos ambientes naturais estão presentes, o que acontece quando ocorre no ambiente urbano a existência de uma vegetação mais adequada (espécies nativas) á região estudada (SCHERER; SCHERER; PETRY; TEIXEIRA 2006; BRUN; LINK; BRUN, 2007; JEBAI, 2009).

A presença em maior quantidade de Passer domesticus, pode ser considerada normal. É uma espécie com características cosmopolitas, conseguindo encontrar seu alimento e abrigo facilmente, não é afetado pela alta urbanização podendo ser encontrado de forma abundante em centros urbanos (EMLEN, 1974, TORGA; FRANCHIN; MARÇAL JÚNIOR, 2007), pois sua dieta é à base de sementes durante a maior parte do ano e de insetos na época de reprodução (SICK, 2001) e ainda se alimenta de rações animais e restos de alimentos humanos.

Columba lívia, assim como Columbina talpacoti e Zenaida auriculata são relatadas como abundantes também nos estudos de Torga; Franchin; Marçal Júnior, 2007, podendo assim como Passer domesticus serem apontados como bioindicadores negativos (EMLEN 1974; AMÂNCIO; SOUZA E MELO, 2008) dada a grande capacidade de sobrevivência em ambientes antrópicos com baixa disponibilidade de recursos naturais.

O fato de Psittacara leucophtalmus ser a segunda espécie em abundância pode estar vinculada ao fato de a mesma ser muita ativa, frequentando diversos locais em curto período de tempo, estabelecendo-se a dúvida se esta espécie poderia ou não ser classificada como uma possível bioindicadora negativa. Psittacara leucophtalmus é encontrada frequentemente nidificando em habitações urbanas, bandos numerosos usam com frequência diversas espécies arbóreas urbanas como dormitório, com a maioria dos indivíduos deslocando-se ao amanhecer para outras áreas para se alimentar, dependendo da época do ano podem permanecer ou não por mais tempo nas cidades durante o período diurno.

Regente Feijó por se tratar de uma cidade pequena, não conta com grandes edifícios, tendo em geral uma arborização bem planejada, onde as espécies de aves possuem acesso livre entre os ambientes pesquisados, há praças que as aves utilizam para se alimentarem devido à 
presença de depósitos de grãos de sementes nas proximidades, e outras elas utilizam para dormir, pelo fato de serem mais arborizadas e tranquilas, sendo estes ambientes os mais propícios para se abrigarem (PAETZOLD; QUEROL, 2008).

Concluímos que em Regente Feijó, as espécies de aves mais adaptadas foram Passer domesticus e Psittacara leucophtalmus. Essa maior presença se deve ao fato de suas dietas estarem à disposição e os ambientes estarem favoráveis a sua sobrevivência. Portanto podemos considerar Passer domesticus como uma espécie bioindicadora negativa de qualidade ambiental com relação ao município de Regente Feijó. Quanto a Psittacara leucophtalmus precisaria de um estudo mais aprofundado, dado ao seu comportamento que a torna uma ave muito ativa.

\section{REFERÊNCIAS}

Amâncio S, Souza VB, Melo C. Columba livia e Pitangus sulphuratus como indicadoras de qualidade ambiental em área urbana. Revista Brasileira de Ornitologia. 2008; v. 16, n. 1, p. 32-37.

Brun FGK; Link D; Brun EJ. O emprego da arborização na manutenção da biodiversidade de fauna em áreas urbanas. Revista da Sociedade Brasileira de Arborização Urbana. 2007; v. 2, n., p. 117127.

CBRO (COMITÊ BRASILEIRO DE REGISTROS ORNITOLÓGICOS) (2014) Listas das aves do Brasil. 11a Edição, 1/1/2014, Disponível em <http://www.cbro.org.br>. Acesso em: 11-agosto de 2014.

Develey PF, Endrigo E. Aves: da grande São Paulo. São Paulo, Aves \& Fotos, 2011.

Endrigo E. Aves: Estado de São Paulo. Aves \& Fotos. São Paulo, 2010.

Donnelly R, Marzluff, JM. Relative importance of habitat quantity, structure, and spatial pattern to birds in urbanizing environments. Urban Ecosystems. 2006; v. 9, p. 99-117.

Emlen JT. An urban bird community in Tucson Arizona derivation, structure, regulation. Condor. 1974; v. 76, p. 184-197.

Jebai GT; Arakaki BR; Silva CAP; Souza AR, Gomes TM; Anjos L. Análise comparativa da densidade de onze passeriformes em duas áreas urbanas em Londrina, norte do Paraná, Brasil. Revista Brasileira de Ornitologia, 2009; v. 17, n 3-4, p. 183-186.

Monteiro, M.P.; Brandão, A. Estrutura da comunidade de aves do "Campus Samambaia" da Universidade Federal de Goiás. Ararajuba. dez.,1995; p.22-26.

Paetzold V, Querol E. Avifauna urbana do município de Uruguaiana, RS, brasil (resultados parciais). Biodiversidade Pampeana ISSN 1679-6179 PUCRS Uruguaiana. jun 2008; v. 6 n. 1, p. 40-45

Philippsen JS; Benedito E; ZawadzkI CH. Species composition and richness of avifauna in an urban area of southern Brazil. Acta Scientiarum. 2010; v. 32, n. 1, p. 55-62. 
Scherer JFM; Scherer AL; Petry MV; Teixeira EC. Estudo da avifauna associada à área úmida situada no Parque Mascarenhas de Moraes, zona urbana de Porto Alegre (RS). Biotemas. 2006; v. 19, n. 1, p. 107-110.

Sick H. Ornitologia Brasileira. Rio de Janeiro: Nova Fronteira, 912 pp. 2001.

Torga K; Franchin AG; Marçal Júnior O. A avifauna em uma seção da área urbana de Uberlândia, MG. Biotemas, 2007; v 20, n 1, p 7-17.

Verner J. Measuring responses of avian communities to habitat manipulation.

Studies in Avian. Biology. 1981; v. 6: p. 534-547.

Votto AP; Gomes-Junior ACF; Bugoni L; Pereira-Junior J. Sazonalidade da avifauna no campus carreiros da fundação Universidade Federal do Rio Grande, Rio Grande do Sul, Brasil. Estudos de Biologia. 2006; v. 28, n. 62, p. 45-55. 